\title{
Le Bon-Pasteur de Québec
}

\section{Thérèse Boucher}

Volume 36, 1969

URI : https://id.erudit.org/iderudit/1007295ar

DOI : https://doi.org/10.7202/1007295ar

Aller au sommaire du numéro

Éditeur(s)

Les Éditions Historia Ecclesiæ Catholicæ Canadensis Inc.

ISSN

0318-6172 (imprimé)

1927-7067 (numérique)

Découvrir la revue

Citer cet article

Boucher, T. (1969). Le Bon-Pasteur de Québec. Sessions d'étude - Société

canadienne d'histoire de l'Église catholique, 36, 57-65.

https://doi.org/10.7202/1007295ar

Tous droits réservés ㄷ Les Éditions Historia Ecclesiæ Catholicæ Canadensis Inc., 1970
Ce document est protégé par la loi sur le droit d'auteur. L'utilisation des services d'Érudit (y compris la reproduction) est assujettie à sa politique d'utilisation que vous pouvez consulter en ligne.

https://apropos.erudit.org/fr/usagers/politique-dutilisation/ 


\section{Le Bon-Pasteur de Québec}

L'intérêt provoqué par l'Exposition universelle de 1967 a déterminé beaucoup de Canadiens à parcourir l'œuvre de Saint-Exupéry intitulée Terre des Hommes. Quelle profondeur de pensée, quel humanisme l'on pressent chez cet auteur.

Le même humanisme haussé par un esprit de foi et d'amour semble avoir été la source de cette entreprise dont on m'a demandé de vous entretenir aujourd'hui, à savoir : le Bon-Pasteur de Québec. Une simple phrase tirée de cet écrivain résume la naissance de cette communauté : "L'homme se découvre quand il se mesure avec l'obstacle."

La fondatrice ne soupçonnait pas le potentiel de richesses que lui avait décerné la Providence... c'est l'œuvre elle-même qui lui révéla la somme de ses possibilités.

Le Bon-Pasteur de Québec est né en terre canadienne, dans la ville même de Québec, le 12 janvier 1850. Il a été établi par Madame Marie Fitzbach-Roy, devenue Mère Marie-du-Sacré-Cœur, et Monsieur le Chevalier Georges-Manly Muir.

Lors de l'érection de la petite société en congrégation religieuse, le 2 février 1856, l'appellation officielle de ses membres fut celle de "Servantes du Cœur Immaculé de Marie». Cependant, le public d'aujourd'hui, comme celui d'hier, lui garde toujours le nom de "Sœurs du Bon-Pasteur ».

Cette institution se distingue de celle du Bon-Pasteur de Montréal par la fondation, mais elle lui ressemble par les œuvres.

Le premier instigateur de l'Euvre du Bon-Pasteur, Monsieur Georges-Manly Muir (1810-1882), avocat de Québec et greffier à l'Assemblée législative, se révèle un membre très actif de la société SaintVincent-de-Paul. Il visite souvent la prison de Québec. Parmi les détenus, il voit des femmes qui, après avoir mené une vie dépravée, se sont rendues coupables des turpitudes les plus lamentables. Il constate aussi que ces pauvres délaissées n'ont pour toute alternative au sortir de leur détention que le retour à leur vie de désordre.

Hanté par le sort de ces malheureuses, cet homme de foi mûrit un projet audacieux mais combien lumineux et charitable : trouver un refuge pour les personnes désireuses de sortir de leurs égarements. 
A ses collègues des conférences Saint-Vincent-de-Paul, l'œuvre apparaît irréalisable. L'apôtre, ardent et tenace, s'adresse à son évêque qui bénit la démarche sociale de ce généreux chrétien, car il y perçoit un appel de la divine Providence.

Monseigneur Pierre-Flavien Turgeon (1787-1867), évêque de Sydime et administrateur du diocèse de Québec, connaît Madame Marie Fitzbach-Roy et discerne en cette femme des qualités exceptionnelles. A

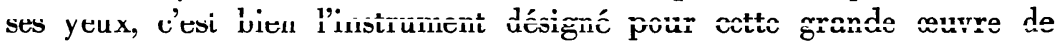
miséricorde.

L'Évêque charge son secrétaire, Monsieur l'abbé Louis Proulx (1804-1871), de transmettre son message. Et le prêtre va présenter à l'âme généreuse la demande de son évêque.

Marie Fitzbach, née à Saint-Vallier de Bellechasse, le 16 octobre 1806, n'avait pas encore deux ans lorsqu'elle perdit son père, Charles Fitzbach. Sa mère, Geneviève Nadeau, épouse par la suite Monsieur Joseph Leclerc, veuf, demeurant à Saint-Charles de Bellechasse, où la jeune Marie ira vivre jusque vers l'âge de 13 ans. La gêne et la privation qui s'étaient établies au foyer l'obligent très tôt à quitter sa petite patrie pour aller prendre un emploi à Québec.

Elle entre au service d'un marchand de la Haute-Ville, Monsieur François-Xavier Roy, qui ne tarde pas à lui confier la gérance de son commerce. Entre temps Marie Fitzbach demande son entrée dans deux monastères québecois, mais la jeune aspirante est refusée, vu sa médiocre santé. Les couvents de Montréal lui font essuyer la même déception. Le Seigneur lui réserve une autre vocation.

Devenu veuf en 1827, Monsieur Roy qui avait su apprécier les qualités exceptionnelles de la jeune employée, tant pour ses affaires personnelles que pour le gouvernement de sa maison, la demande en mariage. C'est en l'église de Cap-Santé, le 17 avril 1828, que Marie Fitzbach unit sa destinée à Monsieur François-Xavier Roy.

Dans son nouvel état de vie, Marie Fitzbach-Roy est à la hauteur de sa tâche : épouse accomplie pour le conjoint malade, mère incomparable pour les deux orphelins Roy et les trois filles que le ciel lui accorde. Mais en dépit d'un dévouement inlassable auprès de Monsieur Roy, celui-ci meurt le 17 septembre 1833.

La famille Roy, avec l'héritage laissé par le défunt, réclame les deux enfants. Madame Marie Fitzbach-Roy n'a que 26 ans. Une nouvelle existence s'ouvre pour elle. Durant dix-sept ans, la Providence va la conduire par des voies mystérieuses vers une destinée inconnue, même insoupçonnée. Éducatrice de ses trois filles, gouvernante au 
presbytère de Saint-Gervais de Bellechasse, elle laisse ciseler son âme par Dieu en vue d'une œuvre plus grande. En 1846 la mort lui enlève Clorinde, la plus jeune de ses filles et, le 22 août 1849, ses deux aînées, Séraphine et Célina, lui annoncent leur décision de se joindre à Mère Mallet, qui arrive à Québec pour fonder un couvent des Sours Grises. Celles-ci quittent leur mère le 8 septembre suivant, et Madame Marie Fitzbach-Roy entre dans une solitude absolue. Elle se voit dans l'obligation d'abandonner sa terre pour occuper une chambre à l'Hospice de la Charité, où ses deux filles s'exercent à la vie religieuse.

C'est ici que Monsieur l'abbé Louis Proulx la rejoint pour lui dire : " Madame, Monseigneur le Coadjuteur a promis à Monsieur Muir de lui faire connaître une personne qui se chargerait de la direction de l'Asile Sainte-Madeleine qu'on se propose d'établir, et il a pensé à vous. Vous sentiriez-vous trop de répugnance à prendre soin des malheureuses qu'on veut y rassembler pour les convertir?"

A l'annonce du message étrange et inattendu, Madame FitzbachRoy, à cause de son indignité personnelle et de la pauvreté de ses moyens, entre dans une agonie indescriptible. Après avoir prié Dieu et consulté ses filles, elle va se prosterner aux pieds de son évêque et dit : "Je suis votre humble servante. Votre volonté sera pour moi celle de Dieu.»

La généreuse réponse de Madame Fitzbach-Roy se concrétise bientôt. C'est le 11 janvier 1850. Accompagnée de Mademoiselle Mary Koegh, elle transporte quelques meubles et de la lingerie au numéro 67, rue Richelieu, à Québec, local loué par la société Saint-Vincent-de-Paul. La tempête fait rage en cette matinée d'hiver, mais le charitable Monsieur Muir est là avec du bois et de l'eau. Aucune provision pour le dîner. Le soir venu, les deux femmes se contentent de pain et de thé. Le lendemain, Monsieur le Grand Vicaire, Charles-Félix Cazeau (18071881), envoie la première recrue à l'Asile Sainte-Madeleine.

Quelques jeunes filles ne tardent pas à venir offrir leurs services à Madame Fitzbach-Roy. Elles vivent laborieuses et retirées, partageant l'unique chambre pendant des nuits d'inquiétude dans une maison mal close. Le matin, c'est le pèlerinage quotidien à la chapelle des Révérends Pères Jésuites, rue Dauphine. Le père Louis Saché, s.j. (1813-1889), devient le premier aumônier (1850-1853) de la petite société.

Madame Marie Fitzbach-Roy œuvre maintenant comme volontaire dans un nouveau secteur de l'Église. Docile à l'Esprit-Saint, elle milite dans son milieu pour répondre à un besoin social et moral.

Le local de la rue Richelieu devient trop restreint. Monsieur l'abbé Louis Proulx, curé de Québec, achète une maison au coin des rues Lachevrotière et Saint-Amable. Les ouvrières déménagent à bras. Elles 
sont trop pauvres pour payer un cocher. Leur passage attire des insultes de la part de ceux qui n'acceptent pas l'œuvre. Les débuts sont pénibles et demandent des sacrifices héroïques aux courageuses fondatrices.

La pauvreté règne dans la maison mais les annales du Bon-Pasteur révèlent les appuis reçus : "Les Révérendes Mères Ursulines et les Messieurs du Séminaire nous permettent d'aller, chaque jour, recueillir les restes de leurs tables dont nous faisons nos repas. Les Mères de l'Hôtel-

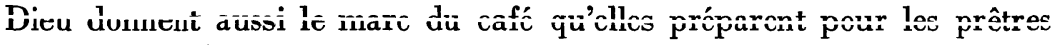
et les élèves du Séminaire.»

Malgré les difficultés rencontrées, la prospérité de l'œuvre naissante étonne tout le monde. Le motif qui a incité Monsieur le Curé de Québec et les membres de la société Saint-Vincent-de-Paul à fixer l'Asile Sainte-Madeleine dans une partie de la Haute-Ville était la possibilité d'y ouvrir une école sous la direction de Madame Fitzbach-Roy. Les enfants qui résident dans le quartier Saint-Louis grandissent dans l'ignorance. Les jeunes filles qui en proviennent ne sont pas admises dans les maisons d'éducation situées à l'intérieur des murs.

La directrice verra donc à leur préparer un local convenable. En attendant, le deuxième étage du hangar est divisé en deux classes et, dès la première semaine, les élèves se présentent au nombre de quatre-vingtdix. Les pauvres sont admises gratuitement. Une contribution mensuelle de douze sous par élève est demandée aux parents capables de payer la scolarité. Le 7 janvier 1851, Sœur Thivierge prend en charge la classe française et Sœur Louisa Wilson enseigne à la jeunesse anglaise. L'établissement de cette école rudimentaire marque un pas de plus du Bon-Pasteur. A la réhabilitation des prisonnières, s'ajoutait l'œuvre de l'éducation des enfants.

L'enseignement a pour promoteur Monsieur Jacques Crémazie (1810-1872), l'un des avocats les plus éminents de Québec, professeur à l'Université Laval et frère aîné de notre poète national, Octave Crémazie. Son expérience pédagogique et ses connaissances juridiques furent toujours gratuitement à la disposition des Sœurs du Bon-Pasteur. C'est ce distingué légiste qui a préparé le projet de loi adopté par le Parlement en 1855 pour l'érection de la communauté en corporation légale.

Jusqu'ici, l'entreprise est apparue d'inspiration laïque. En effet, les deux principales œuvres du Bon-Pasteur: la réhabilitation et l'enseignement sont nées sous l'impulsion du laïcat québecois désireux d'assainir et de promouvoir certaine classe de la société. Monsieur Georges-Manly Muir nous a été révélé comme l'instigateur de la première. Ce brillant avocat, ce grand chrétien, partage avec Madame Fitzbach-Roy la gloire d'avoir établi un asile pour les âmes désireuses de vivre loin du monde qui leur avait été si funeste et de faire pénitence 
sous le regard de Dieu. Monsieur Jacques Crémazie se présente comme le promoteur de la seconde.

Mais l'exercice d'une charité désintéressée ne permet pas de demimesure et finit par exiger un don absolu. Après six années d'engagements apostoliques, l'Association de pieuses laïques connues sous le nom de Sœurs du Bon-Pasteur devient une communauté religieuse.

Samedi, le 2 février 1856, dans la modeste chapelle du Bon-Pasteur, a lieu la première profession religieuse des fondatrices. Elles sont au nombre de sept. Sa Grandeur Monseigneur Charles-François Baillargeon (1797-1870), évêque de Tloa et administrateur du diocèse de Québec, préside la cérémonie. Au même moment, l'Asile du Bon-Pasteur de Québec est canoniquement érigé en communauté religieuse et l'acte d'érection est proclamé par Monsieur l'abbé Edmond Langevin (18241889), secrétaire à l'Archevêché de Québec.

Madame Marie Fitzbach-Roy se nomme désormais Mère Marie-duSacré-Cœur tandis que les Sœurs du Bon-Pasteur reçoivent le titre glorieux de Servantes du Cœur Immaculé de Marie.

La fin des Servantes du Cour Immaculé de Marie, Refuge des pécheurs, est non seulement de s'appliquer à leur propre salut et à leur perfection, avec le secours de la grâce divine, mais de s'employer aussi, de toutes leurs forces, au salut du prochain.

Elles travailleront surtout à ramener à Dieu les pauvres filles pénitentes et à les faire avancer dans les vertus chrétiennes.

Dans la même intention de contribuer au salut des âmes, elles se proposent d'instruire et de former à la piété, les enfants, principalement ceux des pauvres (Constitutions 1856).

Avec les années qui suivent, plusieurs formes de travail social s'intègrent à l'œuvre de réhabilitation des Sœurs du Bon-Pasteur.

L'Hospice de la Miséricorde, fondé dès 1852 par Monsieur l'abbé Joseph Auclair (1813-1887), curé de la cathédrale de Québec et Mademoiselle Marie Métivier (1812-1885), commença sur la rue Couillard.

Pendant une vingtaine d'années quelques filles-mères apportent une faible pension, mais la plupart ne paient rien. Les religieuses du BonPasteur s'occuperont complètement de cette ouvre de Maternité et de Crèche dès 1874. L'allocation du Gouvernement est de $\$ 1,500.00$ dollars par année, c'est-à-dire environ huit dollars par année pour chaque patiente. Ici encore, les religieuses font appel à la charité privée. Des quêtes paroissiales sont organisées par Messieurs les Curés, et les religieuses vont de porte en porte recueillir les aumônes qui paieront la nourriture et le vêtement des pauvres enfants abandonnés.

C'est en 1870 que la Communauté du Bon-Pasteur est appelée à établir une Ecole de Réforme et d'Industrie. Une toute petite maison, 
voisine du couvent sur la rue Saint-Amable, sert de point de départ. En 1876, un édifice de trois étages est construit aux frais de l'institut et permet de recevoir un plus grand nombre de délinquantes. Cette œuvre est déménagée, en 1892, au quartier Saint-Roch, dans les locaux de l'ancien Hôpital de la Marine, devenu Hospice Saint-Charles.

Il faut nommer ici Monsieur Félix Fortier (1811-1890), avocat de grand mérite, qui, par un don généreux, a favorisé l'agrandissement du

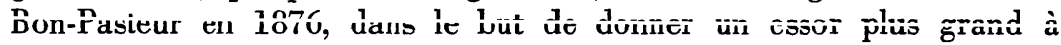
l'œuvre des Madeleines.

Il serait téméraire de vouloir rappeler tous les noms des grands bienfaiteurs qui ont affermi l'œuvre. Nous voulons toutefois signaler les principaux conseillers qui ont orienté la spiritualité du Bon-Pasteur de Québec.

Le révérend Père Louis Saché, s.j., porte à juste titre le nom de fondateur spirituel du Bon-Pasteur de Québec. Cet homme venait lui aussi à l'heure voulue, et il venait avec toutes les qualités requises pour asseoir cette maison sur des bases solides que rien ne pourrait ébranler, et pour lui donner cette abondance de vitalité religieuse nécessaire au fondement de toute communauté.

Le Père Saché initia les fondatrices aux exigences de la vie religieuse. Il prépara les premières constitutions qui furent pendant longtemps le guide de leur vie spirituelle. Ce ministère ne dura que trois ans. Mais l'influence des saints ne se mesure pas à la durée de leur action. Le Père Saché laissait sur l'œuvre du Bon-Pasteur une empreinte impérissable. Il fut remplacé par Monsieur l'abbé J.-Baptiste Ferland (1805-1865), historien très remarquable par ses qualités d'esprit et de cœur, et surtout par ses vertus sacerdotales.

Mais l'ami, le bienfaiteur, l'homme providentiel réservé au BonPasteur, fut Monsieur le Grand Vicaire Charles-Félix Cazeau (1807. 1881).

Le Bon-Pasteur, élevé à la dignité de communauté religieuse, en 1856, prenait une place glorieuse parmi les nombreuses congrégations qui font l'honneur de l'Église du Canada. Pour des nécessités nouvelles, la Providence envoyait un homme nouveau. Sous sa vigoureuse et intelligente impulsion, le Bon-Pasteur connut bientôt des développements remarquables. Monseigneur Cazeau attira sur cette institution la popularité dont il jouissait lui-même. Grâce à l'éclat que sa présence jetait sur la communauté, les vocations vinrent plus confiantes, plus nombreuses et aussi plus persévérantes : car il ne se contentait pas de les faire naître, il les cultivait aussi avec toute la solicitude inspirée par son grand cœur et sa vaste expérience. Grâce à cet essor quantitatif 
autant que qualitatif, les religieuses purent répondre aux appels nombreux lancés à leur dévouement. Les fondations se multiplièrent, mais elles se firent sans hâte, seulement quand la formation religieuse fut complète. Bien pénétrées de l'esprit de leur CEuvre, ces femmes pouvaient quitter avec confiance le berceau de leur vie religieuse et aller au loin sans témérité. Elles gardaient fidèlement les traits essentiels de la religieuse du Bon-Pasteur.

Après avoir été pendant vingt-cinq ans aumônier au Bon-Pasteur de Québec, Monseigneur Cazeau fut remplacé par Monsieur l'abbé AndréAlbert Blais (1824-1919). Homme de compétence reconnue et de culture universelle, il apporte une forme nouvelle aux Constitutions et les fait imprimer avant de quitter le Bon-Pasteur pour l'évêché de Rimouski.

Il nous est impossible de passer sous silence les noms de Nosseigneurs Baillargeon, Dominique et Antoine Racine, du cardinal Taschereau et de nombreux curés des diverses paroisses, pour montrer que le Bon-Pasteur était entouré d'hommes de haut savoir et de vertu éprouvée. Voilà autant de figures qui ont contribué au développement apostolique du Bon-Pasteur.

La vie spirituelle, qui existe au sein de cette congrégation, assure à l'Euvre des proportions grandissantes. Les vocations ne suffisent plus et il faut songer à un nouveau recrutement. Des jeunes filles se présentent comme auxiliaires et désirent vivre dans la Congrégation des Servantes du Coeur Immaculé de Marie.

Il n'est pas alors question de soeurs converses comme il est d'usage dans les ordres anciens. Les jeunes filles sont accueillies par les travaux ménagers. A la recherche d'un moyen terme, le 25 juillet 1874, elles sont groupées dans une fraternité sous le nom de * Tertiaires Dominicaines». Le noviciat d'un an est suivi d'un vœu annuel de chasteté et de stabilité. Ce n'est qu'une promesse de demeurer au BonPasteur et de se dévouer aux CEuvres de l'institution.

Cette nouvelle semence, mise en terre au Bon-Pasteur, germera d'une façon resplendissante. Le noyau se scinde et le fruit mûr se détache de l'arbre en 1887. Une communauté complètement séparée du Bon-Pasteur de Québec a pris naissance : celle des Dominicaines de l'Enfant-Jésus de Québec.

Deux ans plus tôt, le premier septembre 1885, Mère Marie-duSacré-Coeur, Marie Fitzbach-Roy, fondatrice et supérieure du premier institut religieux québecois, était décédée, après avoir eu la consolation de voir grandir et prospérer l'œuvre capitale de sa vie. Elle avait vu, à sa suite, cent quatre-vingt-quinze religieuses assurer la relève du Bon-Pasteur de Québec. 
En 1900, un Te Deum de reconnaissance s'échappe spontanément de tous les cœurs, Te Deum engendré, cinquante ans auparavant, par le fiat de résignation de la vénérée fondatrice.

Ce demi-siècle déroule une série d'événements où le Bon-Pasteur a déployé des merveilles de puissance et d'amour. Trois infatigables ouvrières de la première profession sont encore là pour en retracer la touchante histoire. En cette heure où le passé est présent, c'est la proclamation d'un dévouement pousse jusquä ïhéroïsme.

Sœur Marie-de-Bon-Secours (Séraphine Roy, 1829-1913) et Sœur Saint-Pierre (Célina Roy, 1830-1917), les deux filles de Madame Marie Fitzbach-Roy et religieuses de la Charité, partagent la jubilation du BonPasteur. Quand un demi-siècle a passé sur le faîte d'une institution. il en a consolidé les bases. L'or de son jubilé en proclame la pérennité.

Madame Marie Fitzbach-Roy avait pressenti comme le grand auteur français la vérité de cette assertion : "Être homme, c'est sentir, en posant sa pierre, que l'on contribue à bâtir le monde "(Saint-Exupéry).

Elle a construit un édifice qui ne saurait être ébranlé par le temps, car il tient sur des bases solides d'humilité, de charité et d'attention aux signes des temps. Élevée d'abord pour assurer le redressement de déviations morales et sociales, son œuvre veut favoriser le règne de Dieu et protéger le patrimoine de l'Église.

D'une première maison ont essaimé, pendant plus d'un siècle, des apôtres voués à la sauvegarde du petit, du faible, de la société tout entière. Après avoir donné aux déshérités deux de ses filles, devenues Sœurs de la Charité, Madame Marie Fitzbach-Roy multiplia sur la terre des hommes les ouvrières de leur promotion sociale.

Lorsque l'Église approuve un institut, elle le reconnait comme une école de sainteté. Engendré par un laïcat vraiment conscient de son rôle, le Bon-Pasteur s'est épanoui en une congrégation religieuse qui se doit de témoigner de la vie ecclésiale puisqu'elle est une cellule de l’Église.

Une cellule se renouvelle selon les besoins de l'organisme; devant les appels des temps, le Bon-Pasteur a changé ses structures, mais son dynamisme intérieur en ressort plus ardent, tout de dévouement, de zèle et de charité à la suite de son divin Modèle. Il se garde attentif aux urgences modernes, prêt à découvrir les nouvelles blessures des brebis pour leur offrir encore des prés verdoyants.

Un arbre vigoureux est sorti du grain de sénevé enfoui au cœur du sol québecois en 1850. Autant de maisons filiales, autant de rameaux 
qui couvrent de leur ombre tous ceux qui s'y laissent abriter, pauvres ou moins pauvres, capables de découvrir l'amour et la miséricorde qui élargissait l'humanisme des fondateurs et de voir dans le Bon-Pasteur un rayonnement de l'Église en terre canadienne.

Thérèse Boucher, s.c.i.m.

Maître ès lettres en Sciences religieuses (Histoire), Professeur à la Faculté des Arts de

l'Université Laval. 